\title{
Desenvolvimento de um Modelo Híbrido de Baterias
}

\author{
Luana Fransozi* Airam Sausen Paulo S. Sausen \\ Depto de Ciências Exatas e Engenharias, Mestrado em Modelagem Matemática, Unijuí \\ 98700-000, Ijuí, RS \\ E-mail: luh.fransozi@hotmail.com, airam@unijui.edu.br, sausen@unijui.edu.br
}

\begin{abstract}
RESUMO
Nas últimas décadas novas tecnologias estão sendo desenvolvidas buscando proporcionar melhores condições de vida e conforto a população. Neste contexto, encontram-se os dispositivos móveis que permitem a comunicação entre as pessoas e o acesso rápido a informações em qualquer lugar do mundo. Uma das vantagens destes dispositivos é a mobilidade, a qual é obtida com o auxílio de uma fonte de energia, denominada bateria, que permite manter o aparelho operacional e que está condicionada a um tempo de vida. Define-se por tempo de vida o tempo necessário para atingir um determinado nível de capacidade de carga (i.e., nível de cutoff). A partir do momento em que esse nível é alcançado as reações eletroquímicas cessam e não é mais fornecida energia ao sistema. Por isso, torna-se importante a existência de métodos capazes de predizer o tempo de vida de baterias, assim como representar seu comportamento dinâmico. Uma das formas é através da utilização de modelos matemáticos que descrevem uma situação real apropriando-se da linguagem matemática. Tratando-se de baterias o grande desafio é capturar as características reais das mesmas visto que, há uma grande influência do ambiente interno e externo agindo sobre elas durante o descarregamento. Ao longo dos anos, têm sido desenvolvidos diferentes modelos de baterias, tais como, Modelos Eletroquímicos [2], Modelos de Circuitos Elétricos [1,2], Modelos Estocásticos [2], Modelos Analíticos [2,4] e Modelos Híbridos. Destaca-se que os Modelos Analíticos utilizam um conjunto reduzido de equações, tornando sua implementação simples. O Modelo Analítico de Difusão de Rakhmatov e Vrudhula [4] considera os aspectos físicos das operações de descarga da bateria conseguindo capturar os seus efeitos não-lineares. $O$ processo de difusão unidimensional do modelo é descrito pela Lei de Fick
\end{abstract}

$$
-J(x, t)=D \frac{\partial C(x, t)}{\partial x}
$$

e pela Equação Diferencial Parcial (EDP)

$$
\frac{\partial C(x, t)}{\partial t}=D \frac{\partial^{2} C(x, t)}{\partial^{2} x}
$$

onde $J(x, t)$ é o fluxo das espécies eletroativas em um tempo $t$ e em função de uma distância $x$ do eletrodo, $D$ é a constante de difusão e $C(x, t)$ é a concentração de espécies eletroativas no tempo $t \in[0, L]$ e na distância $x \in[0, w]$. Considerando uma bateria completamente carregada (i.e., $t=0$ ) a concentração de espécies é constante através do comprimento do eletrólito, proporcionando a seguinte condição inicial

$$
C(x, 0)=C^{*} .
$$

De Acordo com a Lei de Faraday, o fluxo de espécies eletroativas na superfície do eletrodo $(x=0)$ é proporcional à corrente $i(t)$ (i.e., carga externa aplicada) e o fluxo na outra extremidade da região de difusão $(x=w)$ é zero. Estas proposições fornecem as seguintes condições de fronteira

$$
\begin{gathered}
\left.D \frac{\partial C(x, t)}{\partial x}\right|_{x=0}=\frac{i(t)}{v F A^{\prime}} \\
\left.D \frac{\partial C(x, t)}{\partial x}\right|_{x=w}=0,
\end{gathered}
$$

onde $A$ é área da superfície do eletrodo, $F$ é a constante de Faraday e $v$ é o número de elétrons envolvidos na reação eletroquímica na superfície do eletrodo.

A partir de manipulações matemáticas obtém-se a equação 


$$
I=\frac{\alpha}{2\left[L\left[1+2 \sum_{n=1}^{\infty}\left(e^{\frac{-\beta^{2} n^{2}}{L}}-\frac{\pi e^{\frac{-\beta^{2} n^{2}}{L}}}{\pi-1+\sqrt{1+\frac{\pi L}{\beta^{2} n^{2}}}}\right)\right]\right.}
$$

que relaciona uma corrente de descarga $I$ com tempo de vida $L$ e os parâmetros empíricos $\alpha$ e $\beta$ que dependem da bateria utilizada, e portanto precisam ser estimados.

Em [3] é apresentado uma análise comparativa de três Modelos Analíticos utilizados para a predição do tempo de vida de baterias, o Modelo Linear, a Lei de Peukert e o Modelo de Difusão de Rakhmatov-Vrudhula. Após realizar um conjunto de simulações computacionais a fim de compará-las com os resultados obtidos a partir de uma plataforma de testes, o autor verificou que o Modelo de Rakhmatov-Vrudhula obteve o melhor desempenho para cargas constantes e cargas variáveis, apresentando um erro médio de 5,71\% e 6,53\% respectivamente. No entanto, os Modelos Analíticos não capturam a tensão e a corrente, características consideradas nos Modelos de Circuitos Elétricos. Os Modelos de Circuitos Elétricos descrevem a bateria na forma de circuito utilizando a combinação de componentes elétricos (fontes, resistores, capacitores e indutores). O Modelo para Predizer Runtime e Características V-I de uma bateria consegue combinar as capacidades transientes dos modelos baseados em Thevenin, as características de corrente alternada dos modelos baseados em Impedância e a informação de tempo de vida dos modelos baseados em Runtime. Como resposta, ele prevê o tempo de vida da bateria, o estado estacionário e a resposta transiente, conseguindo capturar todas as características elétricas e dinâmicas da bateria. As equações que descrevem esse modelo, bem como sua validação na qual obteve um erro médio de $1 \%$ podem ser encontradas em [1]. Em contrapartida, este modelo não captura os efeitos não-lineares presentes no processo de descarga de uma bateria.

Os Modelos Híbridos surgem como uma nova alternativa na predição do tempo de vida de baterias, pois possibilitam o acoplamento de dois modelos com características diferentes. Com isso, é possível obter um novo modelo que possui as vantagens de ambos. A proposta deste trabalho é o desenvolvimento de um modelo matemático a partir da união do Modelo Analítico de Difusão Rakhmatov-Vrudhula com o Modelo de Circuitos Elétricos para Predizer Runtime e Características V-I de uma Bateria. Desta forma, a junção destes dois modelos possibilita a obtenção de um novo modelo, capaz de capturar os efeitos não-lineares e ao mesmo tempo as características dinâmicas do circuito da bateria. O novo modelo será validado utilizando uma plataforma de teste, já construída para a validação de modelos, e baterias de Lítio Íon Polímero. Espera-se que o modelo em desenvolvimento represente satisfatoriamente o processo de descarga de uma bateria mostrando-se acurado.

Palavras-chave: Baterias, Modelos Matemáticos Híbridos, Dispositivos Móveis.

\section{Referências}

[1] M. Chen and G.A. Rincon-Mora, Accurate Electrical Battery Model Capable of Predicting Runtime and I-V Performance, IEEE Transactions on Energy Cconversion, vol. 21, n. 2, pg. 504-511, Junho 2006.

[2] M.R. Jongerden and B.R. Haverkort, Battery Modeling, Thecnical Report in Faculty Electrical Engineering, Mathematics and Computer Science, 2008.

[3] A.V.Oliveira, Análise Comparativa de Metodologias de Estimação de Parâmetros Aplicada a Modelos Analíticos Utilizados na Predição do Tempo de Vida de uma Bateria. Dissertação de Mestrado, DCEEng-Unijuí, 2012.

[4] D.N. Rakhmatov and S.B.K. Vrudhula, An Analytical High-Level Battery Model for Use in Energy Management of Portable Electronic Systems, ICCAD '01: Proceedings of theIEEE/ACM international conference on Computer-aided design, pp.488-493,Piscataway, NJ, USA, 2001. 